\title{
Worst Scenario and Domain Decomposition Methods in Geomechanics
}

\author{
I. Hlaváček ${ }^{\mathrm{a}, \mathrm{b}}$, J. Nedoma ${ }^{\mathrm{a}}$, J. Daněk ${ }^{\mathrm{c}, \mathrm{a}}$ \\ ${ }^{a}$ Institute of Computer Science, Academy of Sciences of the Czech Republic, \\ Pod vodárenskou věž 2, 18207 Prague 8, Czech Republic \\ ${ }^{\mathrm{b}}$ Mathematical Institute, Academy of Sciences of the Czech Republic, Žitná 25, \\ 11567 Prague 1, Czech Republic \\ ${ }^{\mathrm{c}}$ Centre of Applied Mathematics, University of West Bohemia, Univerzitni 8, \\ 30614 Pilsen, Czech Republic
}

\begin{abstract}
In geomechanics there are problems whose investigations lead to solving model problems based on variational formulations. Such problems are frequently formulated by variational inequalities as they physically describe the principle of virtual work in its inequality form. In the first part of the contribution the algorithm for the numerical solution of the discussed variational inequality problem will be investigated. The used parallel algorithm is based on a nonoverlapping domain decomposition method for unilateral contact problem with the given friction and the finite element approach. The conditions of solvability will be presented. In the second part of the contribution a unilateral contact problem with friction and with uncertain input data in quasi-coupled thermo-elasticity is analysed. Method of worst scenario will be applied to find the most "dangerous" admissible input data. The solvability of the corresponding worst scenario (antioptimization) problem will be shortly discussed. Numerical experiments, e.g. a tunnel crossing by an active fault will be presented.
\end{abstract}

Key words: unilateral contact, steady-state heat flow, Coulomb friction, finite element analysis, nonoverlapping domain, decomposition method, uncertain data, worst scenario, reliable solution, geomechanics, radioactive waste repositories, geodynamics

\section{Introduction}

In this paper we will deal with semi-coercive contact problem with friction and uncertain input data in linear quasi-coupled thermo-elasticity. The problem represents extension of problems solved in Nedoma (1987), (1998) for

Preprint submitted to Elsevier Science 1 September 2004 
their application in geomechanics of high level radioactive waste repositories. Such problems are frequently formulated by variational inequalities as they physically describe the principle of virtual work in its inequality form.

The first part of the contribution will deal with numerical solution of a geomechanical problem based on the generalized semi-coercive contact problem with the given friction in quasi-coupled thermo-elasticity for the case that " $s$ " bodies of arbitrary shapes are in mutual contacts and are loaded by external forces. The problem will be formulated as the primary variational inequality problem. The corresponding algorithm, employing properties of modern parallel computers with greater number of processors, will be based on nonoverlapping domain decomposition method.

In the second part of the contribution we will assume that the input data will be also uncertain. By uncertain data we mean input data (physical coefficients, right-hand sides, boundary values, friction, etc.), which cannot be determined uniquely but only in some intervals determined by their measurement errors. The notation reliable solution denotes the worst case among a set of possible solutions, where possibility is given by uncertain input data, and the degree of badness is measured by a criterion-functional (Hlaváček (1999), Hlaváček, Nedoma (2002)). The main goal of our investigation will be to find maximal values of this functional depending on the solution of the problem to be solved. Therefore, we will formulate and analyze a corresponding maximization (worst scenario) problem.

\section{Formulation of the thermo-elastic contact problem}

Let us consider a union $\Omega$ of bounded domains $\Omega^{\iota}, \iota=1, \ldots, s$, with Lipschitz boundaries $\partial \Omega^{\iota}$, occupied by elastic bodies such that $\Omega=\cup_{\iota=1}^{s} \Omega^{\iota} \subset \mathbb{R}^{N}, N \in$ $\{2,3\}$. Let the boundary $\partial \Omega=\cup_{\iota=1}^{s} \partial \Omega^{\iota}$ consist of three disjoint parts $\Gamma_{\tau}, \Gamma_{u}$ and $\Gamma_{c}$, such that $\partial \Omega=\bar{\Gamma}_{\tau} \cup \bar{\Gamma}_{u} \cup \bar{\Gamma}_{c}$.

Assume that $(N-1)$-dimensional measures of $\Gamma_{\tau}, \Gamma_{u}$ and $\Gamma_{c}$ are positive, where $\Gamma_{c}=\cup_{k, l} \Gamma^{k l}, \Gamma^{k l}=\partial \Omega^{k} \cap \partial \Omega^{l}, 1 \leq k, l \leq s, k \neq l$, and $\bar{\Gamma}_{\tau}, \bar{\Gamma}_{u}, \bar{\Gamma}_{c}$ denote the closures in $\partial \Omega$.

We will deal with the following quasi-coupled problem of thermo-elasticity, which consists of a pair of boundary value and contact problems to be solved gradually. 


\subsection{Problem of stationary heat conduction - problem $\mathcal{P}_{1}$}

Let $W^{\iota}$ and $T_{1}$ be given functions. Problem $\mathcal{P}_{1}$ is to find a function of temperature $T=\left(T^{1}, \ldots, T^{s}\right)$ such that

$$
\begin{aligned}
& \frac{\partial}{\partial x_{i}}\left(\kappa_{i j}^{\iota} \frac{\partial T^{\iota}}{\partial x_{j}}\right)+W^{\iota}=0 \quad \text { in } \Omega^{\iota}, 1 \leq \iota \leq s, i, j=1, . ., N \\
& \kappa_{i j} \frac{\partial T}{\partial x_{j}} n_{i}=0 \text { on } \Gamma_{u} \\
& T=T_{1}, \quad \text { on } \Gamma_{\tau}, \\
& T^{k}=T^{l},\left(\kappa_{i j} \frac{\partial T}{\partial x_{j}} n_{i}\right)^{k}+\left(\kappa_{i j} \frac{\partial T}{\partial x_{j}} n_{i}\right)^{l}=0 \quad \text { on } \quad \cup_{k, l} \Gamma^{k l}, 1 \leq k, l \leq s .
\end{aligned}
$$

Throughout the paper we use the summation convention, i.e. a repeated index implies summation from 1 to $\mathrm{N}$. Furthermore, $\mathbf{n}^{k}=\left(n_{i}^{k}\right), i=1, . ., N, 1 \leq$ $k \leq s$, denotes the unit normal with respect to $\partial \Omega^{k}, \mathbf{n}^{k}=-\mathbf{n}^{l}$ on $\Gamma^{k l} ;\left(\kappa_{i j}^{l}\right)$ is the matrix of thermal conductivities. Assume that $\kappa^{\iota}$ are positive definite symmetric matrices,

$$
0<\kappa_{0}^{\iota} \leq \kappa_{i j}^{\iota} \zeta_{i} \zeta_{j}|\zeta|^{-2} \leq \kappa_{1}^{\iota}<+\infty \quad \text { for a.a. } \mathbf{x} \in \Omega^{\iota}, \zeta \in \mathbb{R}^{N}
$$

where $\kappa_{0}^{\iota}, \kappa_{1}^{\iota}$ are constants independent of $\mathbf{x} \in \Omega^{\iota}$. Let $\kappa_{i j}^{\iota} \in L^{\infty}\left(\Omega^{\iota}\right), W^{\iota} \in$ $L^{2}\left(\Omega^{\iota}\right), T_{1} \in H^{1}\left(\Omega^{\iota}\right), T_{1}^{k}=T_{1}^{l}$ on $\cup_{k, l} \Gamma^{k l}$.

Definition 2.1 We say that a function $T$ is a weak solution of problem $\mathcal{P}_{1}$, if $T-T_{1} \in V_{1}$ and

$$
b(T, z)=s(z) \quad \forall z \in V_{1},
$$

where

$$
\begin{gathered}
b(T, z)=\sum_{\iota=1}^{s} \int_{\Omega^{\iota}} \kappa_{i j}^{\iota} \frac{\partial T^{\iota}}{\partial x_{i}} \frac{\partial z^{\iota}}{\partial x_{j}} d \mathbf{x}, \\
s(z)=\sum_{\iota=1}^{s} \int_{\Omega^{\iota}} W^{\iota} z^{\iota} d \mathbf{x}, \\
V_{1}=\left\{z \in W_{1}=\Pi_{\iota=1}^{s} H^{1}\left(\Omega^{\iota}\right) \mid z=0 \text { on } \Gamma_{\tau}, z^{k}=z^{l} \text { on } \cup_{k, l} \Gamma^{k l}\right\} .
\end{gathered}
$$

The formulation (2.5) can be obtained by multiplying equation (2.1) by a test function, integrating by parts over the domain $\Omega^{\iota}$ and using the boundary conditions. 


\subsection{Problem of unilateral contact problem with friction - problem $\mathcal{P}_{2}$}

Let the body forces $\mathbf{F}$, the surface tractions $\mathbf{P}$, boundary displacements $\mathbf{u}_{0}$, elastic coefficients $c_{i j k l}^{\iota}$, coefficients of thermal expansion $\beta_{i j}^{\iota}$ and slip limits $g_{c}^{k l}$, the temperature $T^{\iota}$ and the reference temperature $T_{0}^{\iota}=T_{0}^{\iota}(\mathbf{x})$ be given.

We will deal with the following problem:

Problem $\mathcal{P}_{2}$ : Find the displacement field $\mathbf{u}=\left(u_{i}\right), i=1, . ., N$ in $\Omega$, such that

$$
\begin{gathered}
\frac{\partial}{\partial x_{j}} \tau_{i j}\left(\mathbf{u}^{\iota}, T^{\iota}\right)+F_{i}^{\iota}=0 \quad \text { in } \Omega^{\iota}, 1 \leq \iota \leq s, i=1, \ldots, N, \\
\tau_{i j}\left(\mathbf{u}^{\iota}, T^{\iota}\right)=c_{i j k l}^{\iota} e_{k l}\left(\mathbf{u}^{\iota}\right)-\beta_{i j}^{\iota}\left(T^{\iota}-T_{0}^{\iota}\right) \quad \text { in } \Omega^{\iota}, 1 \leq \iota \leq s, i=1, \ldots, N, \\
\mathbf{u}=\mathbf{u}_{0} \quad \text { on } \Gamma_{u} \\
\tau_{i j}(\mathbf{u}, T) n_{j}=P_{i} \quad \text { on } \Gamma_{\tau}, i=1, \ldots, N, \\
u_{n}^{k}-u_{n}^{l} \leq 0, \tau_{n}^{k} \leq 0,\left(u_{n}^{k}-u_{n}^{l}\right) \tau_{n}^{k}=0 \quad \text { on } \cup_{k, l} \Gamma^{k l}, 1 \leq k, l \leq s, \\
\left|\tau_{t}^{k l}\right| \leq g^{k l} \text { on } \cup_{k, l} \Gamma^{k l}, 1 \leq k, l \leq s, \\
\left|\tau_{t}^{k l}\right|<g^{k l} \Longrightarrow \mathbf{u}_{t}^{k}-\mathbf{u}_{t}^{l}=0 \\
\left|\tau_{t}^{k l}\right|=g^{k l} \Longrightarrow \text { there exists } \vartheta \geq 0 \text { such that } \mathbf{u}_{t}^{k}-\mathbf{u}_{t}^{l}=-\vartheta \tau_{t}^{k l}
\end{gathered}
$$

Here $e_{i j}(\mathbf{u})=\frac{1}{2}\left(\frac{\partial u_{i}}{\partial x_{j}}+\frac{\partial u_{j}}{\partial x_{i}}\right), u_{n}^{k}=u_{i}^{k} n_{i}^{k}, u_{n}^{l}=u_{i}^{l} n_{i}^{k} \quad\left(\right.$ no sum over $k$ or $l$ ), $\mathbf{u}_{t}^{k}=$ $\left(u_{t i}^{k}\right), u_{t i}^{k}=u_{i}^{k}-u_{n}^{k} n_{i}^{k}, \mathbf{u}_{t}^{l}=\left(u_{t i}^{l}\right), u_{t i}^{l}=u_{i}^{l}-u_{n}^{l} n_{i}^{l}, i=1, \ldots, N, \tau_{n}^{k}=\tau_{i j}^{k} n_{i}^{k} n_{j}^{k}$, $\tau_{t}^{k}=\left(\tau_{t i}^{k}\right), \tau_{t i}^{k}=\tau_{i j}^{k} n_{j}^{k}-\tau_{n}^{k} n_{i}^{k}, \tau_{n}^{l}=\tau_{i j}^{l} n_{i}^{l} n_{j}^{l}, \tau_{t}^{l}=\left(\tau_{t i}^{l}\right), \tau_{t i}^{l}=\tau_{i j}^{l} n_{j}^{l}-\tau_{n}^{l} n_{i}^{l}$, $\tau_{t}^{k l} \equiv \tau_{t}^{k}$.

Assume that $c_{i j k l}^{l}$ are positive definite symmetric matrices such that

$$
0<c_{0}^{\iota} \leq c_{i j k l}^{\iota} \xi_{i j} \xi_{k l}|\xi|^{-2} \leq c_{1}^{\iota}<+\infty \quad \text { for a.a. } \mathbf{x} \in \Omega^{\iota}, \xi \in \mathbb{R}^{N^{2}}, \xi_{j i}=\xi_{i j},
$$

where $c_{0}^{\iota}, c_{1}^{\iota}$ are constants independent of $\mathbf{x} \in \Omega^{\iota}$. Let $c_{i j k l}^{\iota} \in L^{\infty}\left(\Omega^{\iota}\right), F_{i}^{\iota} \in$ $L^{2}\left(\Omega^{\iota}\right), P_{i} \in L^{2}\left(\Gamma_{\tau}\right), \beta_{i j}^{\iota} \in L^{\infty}\left(\Omega^{\iota}\right), \mathbf{u}_{0}^{\iota} \in\left[H^{1}\left(\Omega^{\iota}\right)\right]^{N}$. Let coefficients of thermal expansion $\beta_{i j}$ be such that $\beta_{i j}=\beta_{j i}$.

To simplify the formulation of stress-strain relations, the entries of any symmetric $(N \times N)$ matrix $\left\{\tau_{i j}\right\}$ will be denoted by the vector notation $\left\{\tau_{j}\right\}$, $j=1, \ldots, j_{N}$, where $j_{N}=N(N+1) / 2$, as follows:

$$
\begin{gathered}
\tau_{i}=\tau_{i i} \quad \text { for } 1 \leq i \leq N, \tau_{3}=\tau_{12} \quad \text { for } N=2, \\
\tau_{4}=\tau_{23}, \tau_{5}=\tau_{31}, \tau_{6}=\tau_{12} \quad \text { for } N=3 .
\end{gathered}
$$


Likewise, we replace the symmetric matrices $\left(e_{i j}(\mathbf{u})\right),\left(\beta_{i j}\right)$ by vectors $\left\{e_{j}(\mathbf{u})\right\}$, $\left\{\beta_{j}\right\}$. Then the stress-strain relation $(2.7)$ can be rewritten as

$$
\tau_{i}\left(\mathbf{u}^{\iota}, T^{\iota}\right)=\sum_{j=1}^{j_{N}} \mathcal{A}_{i j}^{\iota} e_{j}\left(\mathbf{u}^{\iota}\right)-\beta_{i}^{\iota}\left(T^{\iota}-T_{0}^{\iota}\right), 1 \leq i, j \leq j_{N}, 1 \leq \iota \leq s,
$$

where $\mathcal{A}^{\iota}$ is a symmetric $\left(j_{N} \times j_{N}\right)$ matrix, $\mathcal{A}_{i k}^{\iota} \in L^{\infty}\left(\Omega^{\iota}\right), \iota=1, \ldots, s$.

It is readily seen that

$$
\tau: e \equiv \tau_{i j} e_{i j}=\sum_{i=1}^{N} \tau_{i} e_{i}+2 \sum_{i=N+1}^{j_{N}} \tau_{i} e_{i} .
$$

Therefore, we can write

$$
c_{i j k l}^{\iota} e_{i j} e_{k l}=\sum_{i, j=1}^{j_{N}} B_{i j}^{\iota} e_{i} e_{j},
$$

where $B^{\iota}$ is a symmetric $\left(j_{N} \times j_{N}\right)$ matrix such that

$$
\begin{gathered}
B_{i j}^{\iota}=\mathcal{A}_{i j}^{\iota} \quad \text { for } 1 \leq i, j \leq N, \\
B_{i j}^{\iota}=0 \quad \text { for } 1 \leq i \leq N, N+1 \leq j \leq j_{N}, \\
B_{i j}^{\iota}=2 \mathcal{A}_{i j}^{\iota} \quad \text { for } N+1 \leq i, j \leq j_{N} .
\end{gathered}
$$

Let us denote

$$
\begin{gathered}
W_{1}=\sqcap_{\iota=1}^{s} H^{1}\left(\Omega^{\iota}\right), \quad\|w\|_{W_{1}}=\left(\sum_{\iota \leq s}\left\|w^{\iota}\right\|_{1, \Omega^{\iota}}^{2}\right)^{\frac{1}{2}} \\
W=\sqcap_{\iota=1}^{s}\left[H^{1}\left(\Omega^{\iota}\right)\right]^{N}, \quad\|\mathbf{v}\|_{W}=\left(\sum_{\iota \leq s} \sum_{i \leq N}\left\|v_{i}^{\iota}\right\|_{1, \Omega^{\iota}}^{2}\right)^{\frac{1}{2}} .
\end{gathered}
$$

Assume that the matrices $B^{\iota}$ are positive definite, so that

$$
0<a_{0}^{\iota} \leq \sum_{i, j=1}^{j_{N}} B_{i j}^{\iota} \xi_{i} \xi_{j}|\xi|^{-2} \leq a_{1}^{\iota}<+\infty \quad \text { for a.a. } \mathbf{x} \in \Omega^{\iota}, \xi \in \mathbb{R}^{j_{N}}
$$

where the constants $a_{0}^{\iota}, a_{1}^{\iota}$ are independent of $\mathbf{x} \in \Omega^{\iota}$.

Finally, let us assume that

$\operatorname{meas}_{N-1}\left(\Gamma_{u} \cap \partial \Omega^{\iota}\right)>0$ and $\operatorname{meas}_{N-1}\left(\Gamma_{\tau} \cap \partial \Omega^{\iota}\right)>0 \quad$ for all $\iota=1, \ldots, s$, and let $\mathbf{u}_{0} \in W, T_{0} \in W_{1}, g_{c}^{k l} \in L^{\infty}\left(\Gamma^{k l}\right), \beta_{j}^{\iota} \in L^{\infty}\left(\Omega^{\iota}\right)$. Let us introduce the space of virtual displacements

$$
V=\left\{\mathbf{v} \in W \mid \mathbf{v}=0 \text { on } \Gamma_{u}\right\}
$$

and the set of admissible displacements

$$
K=\left\{\mathbf{v} \in V \mid v_{n}^{k}-v_{n}^{l} \leq 0 \text { on } \cup_{k, l} \Gamma^{k l}\right\}
$$


We shall define a weak solution of the problem $\mathcal{P}_{2}$, which is motivated by the standard procedure: multiply equations (2.6) by a test vector function, integrate by parts over the domain $\Omega$, use the boundary conditions and assume that $\mathbf{u}_{\mathbf{0}}$ satisfies conditions $u_{0 n}^{k}-u_{0 n}^{l}=0$ on $\cup_{k, l} \Gamma^{k l}$.

Definition 2.2 We say that the function $\mathbf{u}$ is a weak solution of problem $\mathcal{P}_{2}$, if $\mathbf{u}-\mathbf{u}_{0} \in K$ and

$$
a(\mathbf{u}, \mathbf{v}-\mathbf{u})+j_{g}(\mathbf{v})-j_{g}(\mathbf{u}) \geq S(\mathbf{v}-\mathbf{u}, T) \quad \forall \mathbf{v} \in \mathbf{u}_{0}+K,
$$

where

$$
\begin{gathered}
a(\mathbf{u}, \mathbf{v})=\sum_{\iota=1}^{s} \int_{\Omega^{\iota}} \sum_{i, j=1}^{3} B_{i j}^{\iota} e_{i}\left(\mathbf{u}^{\iota}\right) e_{j}\left(\mathbf{v}^{\iota}\right) d \mathbf{x}, \\
j_{g}(\mathbf{v})=\sum_{k, l} \int_{\Gamma^{k l}} g^{k l}\left|\mathbf{v}_{t}^{k}-\mathbf{v}_{t}^{l}\right| d s, \\
S(\mathbf{v}, T)=\sum_{\iota=1}^{s} \int_{\Omega^{\iota}}\left(F_{i}^{\iota} v_{i}^{\iota}+\left(T^{\iota}-T_{0}^{\iota}\right) \beta^{\iota}: e\left(\mathbf{v}^{\iota}\right)\right) d \mathbf{x}+\int_{\Gamma_{\tau}} P_{i} v_{i} d s,
\end{gathered}
$$

where the weak solution $T$ of the problem $\mathcal{P}_{1}$ in $S(\mathbf{v}, T)$ is inserted.

\section{Numerical solution and domain decomposition algoritm}

In this section we deal with the elastic part of problem only, as the domain decomposition algorithm for the thermal part of the problem is the standard problem solved in the literature.

\subsection{Formulation of the problem}

We follow the approach proposed by Le Tallec (1994) and group every two subdomains which share a contact area $\Gamma^{k l}$ into a single "nonlinear" subdomain. We use discretization by linear finite elements and the concept of local Schur complements. The resulting nonlinear equation on the interface is solved by successive approximations. For the starting approximation we choose the solution of the linear problem, where the unilateral contact conditions are replaced by the classical bilateral contact conditions without friction.

Let every domain $\bar{\Omega}^{\iota}$ be divided into $J(\iota)$ subdomains $\Omega_{i}^{\iota}, i \leq J(\iota)$. Let us denote $\Gamma_{i}^{\iota}=\partial \Omega_{i}^{\iota} \backslash \partial \Omega^{\iota}, \iota \in\{1, \ldots, s\}, i \in\{1, \ldots, J(\iota)\}$, a part of dividing line and let $\Gamma=\cup_{\iota=1}^{s} \cup_{i=1}^{J(\iota)} \Gamma_{i}^{\iota}$ represent the whole interface boundary. Let us introduce

$$
T^{\iota}=\left\{j \in\{1, \ldots, J(\iota)\}: \bar{\Gamma}_{c} \cap \bar{\Omega}_{j}^{\iota}=\emptyset\right\}, \iota=1, \ldots, s
$$


the set of all indices of subdomains of the domain $\Omega^{\iota}$ which are not adjacent to a contact, and let

$$
\Omega^{* j}=\cup_{[i, \iota] \in \vartheta} \Omega_{i}^{\iota},
$$

where $\vartheta=\left\{[i, \iota]: \partial \Omega_{i}^{\iota} \cap \Gamma_{c} \neq \emptyset\right\}$, represent subdomains in unilateral contact. Suppose that $\Gamma \cap \Gamma_{c}=\emptyset$. Then for the trace operator $\gamma:\left[H^{1}\left(\Omega_{i}^{\iota}\right)\right]^{N} \rightarrow$ $\left[L^{2}\left(\partial \Omega_{i}^{\iota}\right)\right]^{N}$ we have

$$
V_{\Gamma}=\left.\gamma K\right|_{\Gamma}=\left.\gamma V\right|_{\Gamma}
$$

Let $\gamma^{-1}: V_{\Gamma} \in V$ be an arbitrary linear inverse mapping satisfying

$$
\gamma^{-1} \overline{\mathbf{v}}=0 \quad \text { on } \cup_{k, l} \Gamma^{k l} \quad \forall \overline{\mathbf{v}} \in V_{\Gamma}
$$

Let us introduce restrictions $\bar{R}_{i}^{\iota}: V_{\Gamma} \rightarrow \Gamma_{i}^{\iota} ; L_{i}^{\iota}: L^{\iota} \rightarrow \Omega_{i}^{\iota} ; j_{g i}^{\iota}: j_{g}^{\iota} \rightarrow \Gamma^{k l}$; $a_{i}^{\iota}(.,):. a_{i}^{\iota}(.,.) \rightarrow \Omega_{i}^{\iota} ; V\left(\Omega_{i}^{\iota}\right) \rightarrow \Omega_{i}^{\iota}$ and let

$$
V^{0}\left(\Omega_{i}^{\iota}\right)=\left\{\mathbf{v} \in V \mid \mathbf{v}=0 \text { on } \overline{\left(\cup_{\iota=1}^{s} \Omega^{\iota}\right) \backslash \Omega_{i}^{\iota}}\right\}
$$

be the space of functions with zero traces on $\Gamma_{i}^{\iota}$.

Theorem 3.1 A function $\mathbf{u}$ is a solution of a global problem $\mathcal{P}_{2}$, if and only if: its trace $\underline{\mathbf{u}}=\left.\gamma \mathbf{u}\right|_{\Gamma}$ on the interface $\Gamma$ satisfies the condition

$$
\sum_{\iota=1}^{s} \sum_{i=1}^{J(\iota)}\left[a_{i}^{\iota}\left(\mathbf{u}_{i}^{\iota}(\underline{\mathbf{u}}), \gamma^{-1} \underline{\mathbf{w}}\right)-S_{i}^{\iota}\left(\gamma^{-1} \underline{\mathbf{w}}\right)\right]=0 \quad \forall \underline{\mathbf{w}} \in V_{\Gamma}, \underline{\mathbf{u}} \in V_{\Gamma},
$$

and its restrictions $\left.\mathbf{u}_{i}^{\iota}(\mathbf{u}) \equiv \mathbf{u}\right|_{\Omega_{i}^{\iota}}$ satisfy

(i) the condition

$$
\begin{aligned}
a_{i}^{\iota}\left(\mathbf{u}_{i}^{\iota}(\underline{\mathbf{u}}), \boldsymbol{\varphi}_{i}^{\iota}\right) & =S_{i}^{\iota}\left(\boldsymbol{\varphi}_{i}^{\iota}\right) \\
& \forall \boldsymbol{\varphi}_{i}^{\iota} \in V^{0}\left(\Omega_{i}^{\iota}\right), \quad \mathbf{u}_{i}^{\iota}(\underline{\mathbf{u}}) \in V\left(\Omega_{i}^{\iota}\right),\left.\gamma \mathbf{u}_{i}^{\iota}(\underline{\mathbf{u}})\right|_{\Gamma_{i}^{\iota}}=\bar{R}_{i}^{\iota} \underline{\mathbf{u}},
\end{aligned}
$$

for $i \in T^{\iota}, \iota=1, \ldots, s$, and

(ii) the condition

$$
\sum_{[i, \iota] \in \vartheta} a_{i}^{\iota}\left(\mathbf{u}_{i}^{\iota}(\underline{\mathbf{u}}), \boldsymbol{\varphi}_{i}^{\iota}\right)+j_{g}^{\iota}\left(\mathbf{u}_{i}^{\iota}(\underline{\mathbf{u}})+\boldsymbol{\varphi}_{i}^{\iota}\right)-j_{g}^{\iota}\left(\mathbf{u}_{i}^{\iota}(\underline{\mathbf{u}})\right) \geq \sum_{[i, \iota] \in \vartheta} S_{i}^{\iota}\left(\boldsymbol{\varphi}_{i}^{\iota}\right)
$$

for all $\boldsymbol{\varphi} \equiv\left(\boldsymbol{\varphi}_{i}^{\iota},[i, \iota] \in \vartheta\right), \boldsymbol{\varphi}_{i}^{\iota} \in V^{0}\left(\Omega_{i}^{\iota}\right)$, and such that

$$
\mathbf{u}+\boldsymbol{\varphi} \in K,\left.\quad \gamma \mathbf{u}_{i}^{\iota}(\underline{\mathbf{u}})\right|_{\Gamma_{i}^{\iota}}=\bar{R}_{i}^{\iota} \underline{\mathbf{u}} \quad \text { for }[i, \iota] \in \vartheta .
$$

For the proof see Daněk, Hlaváček, Nedoma (2004). 


\subsection{The Schur complements and the linearized problem}

The aim of this subsection is to analyze in detail the condition (3.5) and to employ it for numerical computation of problem $\mathcal{P}_{2}$. We will introduce the concept of the local Schur complement.

Let us denote $V_{i}^{\iota}=\left\{\left.\gamma \mathbf{v}\right|_{\Gamma_{i}^{\iota}} \mid \mathbf{v} \in K\right\}=\left\{\left.\gamma \mathbf{v}\right|_{\Gamma_{i}^{\iota}} \mid \mathbf{v} \in V\right\}$

and define a particular case of the restriction of the inverse mapping $\left.\gamma^{-1}()\right|_{.\Omega_{i}^{\iota}}$ by

$$
\left\{\begin{array}{cc}
\operatorname{Tr}_{i \iota}^{-1}: V_{i}^{\iota} \rightarrow V\left(\Omega_{i}^{\iota}\right), & \left.\gamma\left(\operatorname{Tr}_{i \iota}^{-1} \underline{\mathbf{u}}_{i}^{\iota}\right)\right|_{\Gamma_{i}^{\iota}}=\underline{\mathbf{u}}_{i}^{\iota}, \\
& i=1, \ldots, J(\iota), \iota=1, \ldots, s, \\
a_{i}^{\iota}\left(\operatorname{Tr}_{i \iota}^{-1} \underline{\mathbf{u}}_{i}^{\iota}, \mathbf{v}_{i}^{\iota}\right)=0 \quad \forall \mathbf{v}_{i}^{\iota} \in V^{0}\left(\Omega_{i}^{\iota}\right), \operatorname{Tr}_{i \iota}^{-1} \underline{\mathbf{u}}_{i}^{\iota} \in V\left(\Omega_{i}^{\iota}\right), \\
\text { for } i \in T^{\iota}, \iota=1, \ldots, s .
\end{array}\right.
$$

For $[i, \iota] \in \vartheta$ we complete the definition by the boundary condition (3.4), i.e.

$$
\operatorname{Tr}_{i \iota}^{-1} \underline{\mathbf{u}}_{i}^{\iota}=0 \text { on } \cup_{k, l} \Gamma^{k l} .
$$

Definition 3.1 By the local Schur complement for $i \in T^{\iota}$ it is meant the operator $\mathcal{S}_{i}^{\iota}: V_{i}^{\iota} \rightarrow\left(V_{i}^{\iota}\right)^{*}$ defined by

$$
\left\langle\mathcal{S}_{i}^{\iota} \overline{\mathbf{u}}_{i}^{\iota}, \overline{\mathbf{v}}_{i}^{\iota}\right\rangle=a_{i}^{\iota}\left(\operatorname{Tr}_{i \iota}^{-1} \overline{\mathbf{u}}_{i}^{\iota}, \operatorname{Tr}_{i \iota}^{-1} \overline{\mathbf{v}}_{i}^{\iota}\right) \quad \forall \overline{\mathbf{u}}_{i}^{\iota}, \overline{\mathbf{v}}_{i}^{\iota} \in V_{i}^{\iota}
$$

and in the matrix form by

$$
\mathcal{S}_{i}^{\iota} \overline{\mathbf{U}}_{i}^{\iota}=\left(\bar{A}_{i \iota}-\bar{B}_{i \iota}^{T} \stackrel{\circ}{A_{i \iota}^{-1}} B_{i \iota}\right) \overline{\mathbf{U}}_{i}^{\iota}
$$

where

$$
A_{i \iota}=\left(\begin{array}{cc}
\stackrel{\circ}{A}_{i \iota} & B_{i \iota} \\
B_{i \iota}^{T} & \bar{A}_{i \iota}
\end{array}\right), \mathbf{U}_{i}^{\iota}=\left(\begin{array}{c}
\stackrel{\circ}{\iota}_{i} \\
\overline{\mathbf{U}}_{i}^{\iota}
\end{array}\right),
$$

where the nodes of $\overline{\mathbf{U}}_{i}^{\iota}$ belong to $\Gamma_{i}^{\iota}$ and the internal degrees of freedom are $\mathbf{\mathbf { U }}_{i}$.

For subdomains which are in contact we will define a common local Schur complement as follows:

Definition 3.2 The common local Schur complement for the union $\Omega_{i}^{k} \cup \Omega_{j}^{l}$ (where $\Gamma_{c}^{k l} \subset \Gamma_{c}$ and $[i, k] \in \vartheta,[j, l] \in \vartheta$ ) is the operator

$$
\mathcal{S}^{k l}:\left(V_{i}^{k} \times V_{j}^{l}\right) \rightarrow\left(V_{i}^{k} \times V_{j}^{l}\right)^{*}=\left(V_{i}^{k}\right)^{*} \times\left(V_{j}^{l}\right)^{*}
$$

defined by the relation

$$
\begin{aligned}
&\left\langle\mathcal{S}^{k l}\left(\overline{\mathbf{y}}_{i}^{k}, \overline{\mathbf{y}}_{j}^{l}\right),\left(\overline{\mathbf{v}}_{i}^{k}, \overline{\mathbf{v}}_{j}^{l}\right)\right\rangle=a_{i}^{k}\left(\mathbf{u}_{i}^{k}\left(\overline{\mathbf{y}}_{i}^{k}\right), \operatorname{Tr}_{i k}^{-1} \overline{\mathbf{v}}_{i}^{k}\right)+ a_{j}^{l}\left(\mathbf{u}_{j}^{l}\left(\overline{\mathbf{y}}_{j}^{l}\right), \operatorname{Tr}_{j l}^{-1} \overline{\mathbf{v}}_{j}^{l}\right) \\
& \forall\left(\mathbf{v}_{i}^{k}, \overline{\mathbf{v}}_{j}^{l}\right) \in V_{i}^{k} \times V_{j}^{l}
\end{aligned}
$$


where $\operatorname{Tr}_{i k}^{-1}$ and $\operatorname{Tr}_{j l}^{-1}$ are defined by means of (3.9) and (3.10) and $\mathbf{u}_{i}^{k}\left(\overline{\mathbf{y}}_{i}^{k}\right)$, $\mathbf{u}_{j}^{l}\left(\overline{\mathbf{y}}_{j}^{l}\right)$ denote the solution of the problem (3.7).

The condition (3.5) can be expressed by means of local Schur complements. Then we have

Lemma 3.2 The trace $\overline{\mathbf{u}}=\left.\gamma \mathbf{u}\right|_{\Gamma}$ of the weak solution satisfies the following condition

$$
\begin{gathered}
\sum_{\iota=1}^{s} \sum_{i \in T^{\iota}}\left\langle\mathcal{S}_{i}^{\iota} \overline{\mathbf{u}}_{i}^{\iota}, \overline{\mathbf{v}}_{j}^{\iota}\right\rangle+\sum_{k, l}\left\langle\mathcal{S}^{k l}\left(\overline{\mathbf{u}}_{i}^{k}, \overline{\mathbf{u}}_{j}^{l}\right),\left(\overline{\mathbf{v}}_{i}^{k}, \overline{\mathbf{v}}_{j}^{l}\right)\right\rangle=\sum_{\iota=1}^{s} \sum_{i=1}^{J(\iota)} L_{i}^{\iota}\left(\operatorname{Tr}_{i \iota}^{-1} \overline{\mathbf{v}}_{i}^{\iota}\right) \\
\forall \overline{\mathbf{v}} \in V_{\Gamma},[i, k] \in \vartheta,[j, l] \in \vartheta, \Gamma^{k l} \subset \Gamma_{c},
\end{gathered}
$$

where $\overline{\mathbf{v}}_{i}^{\iota}=\bar{R}_{i}^{\iota} \overline{\mathbf{v}}, \overline{\mathbf{u}}_{i}^{\iota}=\bar{R}_{i}^{\iota} \overline{\mathbf{u}}$.

Then we will solve the equation (3.15) on the interface $\Gamma$ in the dual space $\left(V_{\Gamma}\right)^{*}$. We rewrite (3.15) into the following form

$$
\mathcal{S}_{0} \overline{\mathbf{U}}+\mathcal{S}_{C O N} \overline{\mathbf{U}}=\mathcal{F}
$$

where

$$
\begin{aligned}
& \mathcal{S}_{0}=\sum_{\iota=1}^{s} \sum_{i \in T^{\iota}}\left(\bar{R}_{i}^{\iota}\right)^{T} \mathcal{S}_{i}^{\iota} \bar{R}_{i}^{\iota}, \quad \mathcal{S}_{C O N}=\sum_{k, l} \bar{R}_{k l}^{T} \mathcal{S}^{k l} \bar{R}_{k l}, \\
& \mathcal{F}=\sum_{\iota=1}^{s} \sum_{i=1}^{J(\iota)}\left(\bar{R}_{i}^{\iota}\right)^{T}\left(T r_{i \iota}^{-1}\right)^{T} \mathcal{S}_{i}^{\iota}
\end{aligned}
$$

and $\bar{R}_{k l}(\overline{\mathbf{u}})=\left(\bar{R}_{i}^{k}(\overline{\mathbf{u}}), \bar{R}_{j}^{l}(\overline{\mathbf{u}})\right)^{T}, \overline{\mathbf{u}} \in V_{\Gamma},[i, k] \in \vartheta,[j, l] \in \vartheta, \Gamma^{k l} \subset \Gamma_{c}$.

Equation (3.16) will be solved by successive approximations, because the operators $\mathcal{S}^{k l}$ and therefore $\mathcal{S}_{C O N}$ are nonlinear. We choose a suitable initial approximation $\mathbf{U}^{0}$, for instance the solution of the global primal problem, where the boundary conditions on $\Gamma_{c}$ are replaced by the linear "classical" bilateral conditions (which correspond with $g^{k l} \equiv 0$ and $j_{g}(\mathbf{u}) \equiv 0$ )

$$
u_{n}^{k}-u_{n}^{l}=0, \tau_{t}^{k l}=0 \quad \text { on } \Gamma_{c 0} \equiv \cup_{k, l} \Gamma_{0}^{k l}
$$

where $\Gamma_{0}^{k l}$ are parts of $\Gamma^{k l}$, meas $\Gamma_{0}^{k l}>0$, chosen a priori (e.g. for example $\left.\Gamma_{0}^{k l}=\Gamma^{k l}\right)$. On $\Gamma^{k l} \backslash \Gamma_{0}^{k l}$ we consider homogeneous conditions of zero surface load $P_{j}^{k}=P_{j}^{l}=0, j=1, \ldots, N$.

Then we replace the set $K$ by $K^{0}=\left\{\mathbf{v} \in V \mid v_{n}^{k}-v_{n}^{l}=0\right.$ on $\left.\cup_{k, l} \Gamma_{0}^{k l}\right\}$ and therefore, we will solve the following problem

$$
\mathbf{u}^{0}=\arg \min _{\mathbf{v} \in K^{0}}\left(\frac{1}{2} a(\mathbf{v}, \mathbf{v})-S(\mathbf{v})\right)
$$

and we set $\overline{\mathbf{U}}^{0}=\left.\gamma \mathbf{u}^{0}\right|_{\Gamma}$. The auxiliary problem (3.19) represents a linear elliptic boundary value problem of a system of " $s$ " elastic bodies with bilateral contact and it can be solved by the domain decomposition method again. 


\subsection{Solution of the auxiliary problem}

Instead of (2.15) we will solve the variational equation for $\mathbf{u}^{0} \in K^{0}$ :

$$
a\left(\mathbf{u}^{0}, \mathbf{v}\right)=S(\mathbf{v}) \quad \forall \mathbf{v} \in K^{0} .
$$

Thus an analogue of Theorem 3.1 can be derived, where the condition (3.7) is replaced by the corresponding variational equality and where a mapping $\gamma_{0}^{-1}: V_{\Gamma} \rightarrow V$ satisfies conditions $\left(\gamma_{0}^{-1} \overline{\mathbf{v}}\right)_{n}^{k}-\left(\gamma_{0}^{-1} \overline{\mathbf{v}}\right)_{n}^{l}=0$ on $\cup_{k, l} \Gamma_{0}^{k l}$.

We introduce operators of Schur complements. For $i \in T^{\iota}, \iota=1, \ldots, s$, we define the mappings $\operatorname{Tr}_{i \iota}^{-1}$ according to (3.9) and the local Schur complemenents $\mathcal{S}_{i}^{0 \iota}$ by (3.11).

Definition 3.3 The common local Schur complement for the union $\Omega_{i}^{k} \cup \Omega_{j}^{l}$, where $\Gamma_{0}^{k l} \subset \Gamma_{c}$ and $[i, k] \in \vartheta,[j, l] \in \vartheta$,

$$
\mathcal{S}^{0 k l}:\left(V_{i}^{k} \times V_{j}^{l}\right) \rightarrow\left(V_{i}^{k}\right)^{*} \times\left(V_{j}^{l}\right)^{*}
$$

is defined by the following relation

$$
\begin{array}{r}
\left\langle\mathcal{S}^{0 k l}\left(\overline{\mathbf{u}}_{i}^{0 k}, \mathbf{u}_{j}^{0 l}\right),\left(\overline{\mathbf{v}}_{i}^{k}, \overline{\mathbf{v}}_{j}^{l}\right)\right\rangle=a_{i}^{k}\left(\mathbf{u}_{i}^{k}\left(\overline{\mathbf{u}}_{i}^{k}\right), \operatorname{Tr}_{i k}^{-1} \overline{\mathbf{v}}_{i}^{k}\right)+a_{j}^{l}\left(\mathbf{u}_{j}^{l}\left(\overline{\mathbf{u}}_{j}^{l}\right), \operatorname{Tr}_{j l}^{-1} \mathbf{v}_{j}^{l}\right) \\
\forall\left(\overline{\mathbf{v}}_{i}^{k}, \overline{\mathbf{v}}_{j}^{l}\right) \in V_{i}^{k} \times V_{j}^{l},
\end{array}
$$

where $\operatorname{Tr}_{i k}^{-1}$ and $\operatorname{Tr}_{j l}^{-1}$ are defined by means of $\left(\operatorname{Tr}_{i k}^{-1} \overline{\mathbf{v}}_{i}^{k}\right)_{n}-\left(\operatorname{Tr}_{j l}^{-1} \overline{\mathbf{v}}_{j}^{k}\right)_{n}=0$ on $\Gamma_{0}^{k l}$ and

$$
\begin{aligned}
& a_{i}^{k}\left(\operatorname{Tr}_{i k}^{-1} \overline{\mathbf{v}}_{i}^{k}, \mathbf{w}_{i}^{k}\right)+a_{j}^{l}\left(\operatorname{Tr}_{j l}^{-1} \overline{\mathbf{v}}_{j}^{l}, \mathbf{w}_{j}^{l}\right)=0 \quad \forall \mathbf{w}_{i}^{k} \in V^{0}\left(\Omega_{i}^{k}\right), \mathbf{w}_{j}^{l} \in V^{0}\left(\Omega_{j}^{l}\right) \\
& \text { such that }\left(\mathbf{w}_{i}^{k}\right)_{n}-\left(\mathbf{w}_{j}^{l}\right)_{n}=0 \quad \text { on } \Gamma_{0}^{k l} .
\end{aligned}
$$

A global Schur complement $\mathcal{S}$ is defined by

$$
\mathcal{S}=\mathcal{S}_{0}+\sum_{k, l}\left(\bar{R}_{k l}\right)^{T} \mathcal{S}^{0 k l} \bar{R}_{k l}
$$

where $\mathcal{S}_{0}$ is defined in (3.17). and $\mathcal{S}^{0 k l}$ by (3.21), (3.22).

Then the condition coresponding to (3.15) of the auxiliary problem on the interface implies the equation

$$
\mathcal{S} \overline{\mathbf{U}}=\mathcal{F} \quad \text { in the dual space }\left(V_{\Gamma}\right)^{*}
$$

To solve problem (3.24) the method of preconditioned conjugate gradients can be used. In Daněk, Hlaváček, Nedoma (2004) the so-called Neumann-Neumann preconditioner is derived. 


\subsection{Successive approximation method and its convergence}

Recall that we have to solve the problem (3.16) by successive approximations. Now $\overline{\mathbf{U}}^{0}$ is the solution of the auxiliary problem, i.e. $\overline{\mathbf{U}}^{0}=\left.\gamma \mathbf{u}^{0}\right|_{\Gamma}$, where $\mathbf{u}^{0}$ is a solution of problem (3.19). The next approximations $\overline{\mathbf{U}}^{k}, k=1,2, \ldots$, we find as the solution of the following linear problem

$$
\mathcal{S}_{0} \overline{\mathbf{U}}^{k}=\mathcal{F}-\mathcal{S}_{C O N} \overline{\mathbf{U}}^{k-1}, k=1,2, \ldots
$$

To solve problem (3.25), we use again the method of preconditioned conjugate gradients with new "reduced" preconditioner of the Neumann - Neumann type (see Daněk, Hlaváček, Nedoma (2004)).

Definition 3.4 We define "injection operators"

$$
\stackrel{\circ}{D_{i}}: V_{i}^{\iota} \rightarrow V_{\Gamma}, \quad \iota=1, \ldots, s \text { and } i \in T^{\iota}
$$

by the following relation. For the nodes on $\Gamma_{i}^{k} \cup \Gamma_{j}^{l}\left(\Gamma^{k l} \subset \Gamma_{c},[i, k] \in \vartheta,[j, l] \in \vartheta\right)$

$$
\begin{gathered}
\stackrel{\circ}{D}_{i}^{\iota} \mathbf{v}\left(P_{m}\right)=\underline{\mathbf{v}}\left(P_{n}\right) \quad \text { if } P_{n} \in \Gamma_{i}^{k} \cup \Gamma_{j}^{l}, \\
\stackrel{\circ}{D} \iota_{i} \underline{\mathbf{v}}\left(P_{m}\right)=\underline{\mathbf{v}}\left(P_{n}\right) \rho_{i}^{\iota} / \rho^{T}
\end{gathered}
$$

if the $m$-th degree of freedom corresponds with the $n$-th degree of $V_{i}^{\iota}$ and $P_{n} \notin \Gamma_{i}^{k} \cup \Gamma_{j}^{l}$ and $\stackrel{\circ}{D_{i}} \mathbf{v}\left(P_{m}\right)=0 \quad$ in the remaining cases.

Here $\rho_{i}^{\iota}$ denotes the local measure of stiffness of the subdomain $\Omega_{i}^{\iota}$ (e.g. the average of the Young modulus) and

$$
\varrho_{T}=\sum_{P_{l} \in \bar{\Omega}_{j}^{\iota}} \varrho_{j}^{\iota}
$$

is the sum of $\rho_{j}^{\iota}$ over all subdomains $\bar{\Omega}_{j}^{\iota}$, which contain the point $P_{l}$.

Let us realize that the kernel

$$
\mathcal{Z}_{i}^{\iota}=\operatorname{Ker} A_{i \iota}, \quad \iota=1, \ldots, s, i \in T^{\iota}
$$

may contain nonzero elements, i.e. displacements of a rigid body $\Omega_{i}^{\iota}$. Therefore, we introduce the orthogonal complement of the kernel $\mathcal{Z}_{i}^{\iota}$ in the space $V\left(\Omega_{i}^{\iota}\right)$, so that

$$
Q\left(\Omega_{i}^{\iota}\right) \oplus \operatorname{Ker} A_{i \iota}=V\left(\Omega_{i}^{\iota}\right)
$$

Let us define the "coarse" reduced space of traces

$$
V_{0 H}=\sum_{\iota=1}^{s} \sum_{i \in T^{\iota}} \stackrel{\circ}{D}_{i}^{\iota} \gamma \mathcal{Z}_{i}^{\iota}
$$

and a linear set $V_{0 H}^{\perp} \in\left(V_{\Gamma}\right)^{*}$ of functionals by the relation

$$
\mathbf{S} \in V_{0 H}^{\perp} \Leftrightarrow\langle\mathbf{S}, \mathbf{z}\rangle=0 \quad \forall \mathbf{z} \in V_{0 H}
$$


The set $V_{0 H}^{\perp}$ will be used for starting values of the preconditioned conjugate gradients algorithm. Now we will analyze the convergence of the method of sucessive approximation (3.25), to the solution of the original problem (3.16) in the space $\left(V_{\Gamma}\right)^{*}$.

To this end, we introduce a seminorm and a norm.

Definition 3.5 Let $\mathbb{H}_{0}$ be an orthogonal complement of the subspace $V_{0 H}$ in $V_{\Gamma}$. Let us introduce a seminorm

$$
\left|\bar{R}_{c \underline{\mathbf{v}}}\right|_{\vartheta}=\left(\sum_{k, l}\left[a_{i}^{k}\left(\operatorname{Tr}_{i k}^{-1} \bar{R}_{i}^{k} \underline{\mathbf{v}}, \operatorname{Tr}_{i k}^{-1} \bar{R}_{i}^{k} \underline{\mathbf{v}}\right)+a_{j}^{l}\left(\operatorname{Tr}_{j l}^{-1} \bar{R}_{j}^{l} \underline{\mathbf{v}}, \operatorname{Tr}_{j l}^{-1} \bar{R}_{j}^{l} \mathbf{\mathbf { v }}\right)\right]\right)^{\frac{1}{2}}
$$

where $\Gamma^{k l} \subset \Gamma_{c},[i, k] \in \vartheta$ and $[j, l] \in \vartheta$.

Lemma 3.3 The expression

$$
\|\underline{\mathbf{u}}\|_{Q}^{2}=\left\langle\mathcal{S}_{0} \underline{\mathbf{u}}, \underline{\mathbf{u}}\right\rangle
$$

defines a norm in $\mathbb{H}_{0}$.

Definition 3.6 Let a mapping $T: \mathbb{H}_{0} \rightarrow \mathbb{H}_{0}$ be defined by the relation

$$
\left\langle\mathcal{S}_{0}(T \underline{\mathbf{y}}), \underline{\mathbf{v}}\right\rangle=\left\langle\mathcal{F}-\mathcal{S}_{C O N}(\underline{\mathbf{y}}), \underline{\mathbf{v}}\right\rangle \quad \forall \underline{\mathbf{v}} \in \mathbb{H}_{0} .
$$

Assumption 3.4 Let a constant $\beta$ exist such that

$$
\left|\bar{R}_{c} \underline{\mathbf{u}}\right|_{\vartheta} \leq \beta\|\underline{\mathbf{u}}\|_{Q} \quad \forall \underline{\mathbf{u}} \in \mathbb{H}_{0} .
$$

Lemma 3.5 If assumption 3.4 is satisfied, the mapping $T$ is well-defined, i.e. for all $\underline{\mathbf{y}} \in \mathbb{H}_{0}$ there exists a unique element $T \underline{\mathbf{y}} \in \mathbb{H}_{0}$, satisfying (3.35).

For the proof see Daněk, Hlaváček, Nedoma (2004).

Theorem 3.6 Let the assumption 3.4 hold. Then

$$
\|T(\underline{\mathbf{y}})-T(\underline{\mathbf{w}})\|_{Q} \leq 2 \beta^{2}\|\underline{\mathbf{y}}-\underline{\mathbf{w}}\|_{Q} \quad \underline{\mathbf{y}}, \underline{\mathbf{w}} \in \mathbb{H}_{0} .
$$

For the proof see Daněk, Hlaváček, Nedoma (2004).

Corollary 3.7 Let the assumption 3.4 hold with $\beta<\sqrt{2} / 2$. Then the mapping $T$ is contractive on $\mathbb{H}_{0}$. The successive approximations (3.25) converge to a 
fixed point of the mapping $T$, which represents a solution $\underline{\mathbf{U}}$ of the equation (3.16). The following error estimate holds

$$
\left\|\underline{\mathbf{U}}^{k}-\underline{\mathbf{U}}\right\|_{Q} \leq\left(2 \beta^{2}\right)^{k}\left(1-2 \beta^{2}\right)^{-1}\left\|\underline{\mathbf{U}}^{0}-T \underline{\mathbf{U}}^{0}\right\|_{Q}, \quad k=1,2, \ldots
$$

for any $\underline{\mathbf{U}}^{0} \in \mathbb{H}_{0}$.

For the proof see Nečas, Hlaváček $(1981, \S 11.7)$.

Remark 3.1 The assumption 3.4 with $\beta<\sqrt{2} / 2$ is fulfilled if the union $\cup_{[i, \imath] \in \vartheta} \Omega_{i}^{\iota}$ of subdomains, adjacent to the contact boundary $\Gamma_{c}$, is "small" with regard to the union of remaining subdomains and if the triangulation of every $\Omega_{i}^{\iota},[i, \iota] \in \vartheta$ is sufficiently fine near $\Gamma_{i}^{\iota}$.

\section{Worst scenario problem for uncertain input data}

\subsection{Sets of uncertain input data}

Let us assume that the input data

$$
A=\left\{B^{\iota}, \kappa^{\iota}, W^{\iota}, T_{1}, \mathbf{F}^{\iota}, \beta^{\iota}, \mathbf{P}, \mathbf{u}_{0}, g^{k l}, \iota=1, \ldots, s, \forall k, l\right\}
$$

are uncertain. Let the only available information about them be that they belong to some sets of admissible data, i.e.,

$A \in U_{a d} \Leftrightarrow B^{\iota} \in U_{a d}^{B^{\iota}}, \kappa^{\iota} \in U_{a d}^{\kappa^{\iota}}, W^{\iota} \in U_{a d}^{W^{\iota}}, T_{1} \in U_{a d}^{T_{1}}, \mathbf{F}^{\iota} \in U_{a d}^{F^{\iota}}, \beta^{\iota} \in U_{a d}^{\beta^{\iota}}$, $\mathbf{P} \in U_{a d}^{P}, \mathbf{u}_{0} \in U_{a d}^{u_{0}}, g^{k l} \in U_{a d}^{g^{k l}}$.

Assume that all the bodies $\Omega^{\iota}$ are piecewise homogeneous, so that partitions of $\bar{\Omega}^{\iota}$ exist such that

$$
\begin{gathered}
\bar{\Omega}^{\iota}=\cup_{j=1}^{\bar{j}_{\iota}} \bar{\Omega}_{j}^{\iota}, \quad \Omega_{j}^{\iota} \cap \Omega_{k}^{\iota}=\emptyset \quad \text { for } j \neq k, 1 \leq \iota \leq s, \\
\Gamma^{k l}=\cup_{q=1}^{Q_{k l}} \bar{\Gamma}_{q}^{k l}, \quad \Gamma_{q}^{k l} \cap \Gamma_{p}^{k l}=\emptyset \quad \text { for } q \neq p, \forall k, l
\end{gathered}
$$

and let the data $B^{\iota}, \kappa^{\iota}, \mathbf{F}^{\iota}, W^{\iota}, \beta^{\iota}$ be piecewise constant with respect to the partition (4.1).

Let us denote

$$
\begin{gathered}
\Gamma_{u} \cap \partial \Omega^{\iota}=\Gamma_{u}^{\iota}, \iota=1, \ldots, s, \\
\Gamma_{\tau} \cap \partial \Omega^{\iota}=\Gamma_{\tau}^{\iota}, \iota \leq s .
\end{gathered}
$$


We define the sets of admissible matrices:

$$
\begin{aligned}
U_{a d}^{B^{\iota}}=\{ & \left(j_{N} \times j_{N}\right) \text { symmetric matrices } B^{\iota}: \\
& \left.\underline{B}_{i k}^{\iota}(j) \leq\left. B_{i k}\right|_{\Omega_{j}^{\iota}}=\text { const. } \leq \bar{B}_{i k}^{\iota}(j), j \leq \bar{j}_{\iota}, i, k=1, \ldots, j_{N}\right\}
\end{aligned}
$$

where $\underline{B}^{\iota}(j)$ and $\bar{B}^{\iota}(j)$ are given $\left(j_{N} \times j_{N}\right)$ symmetric matrices, $\iota=1, \ldots, s$. Assume that positive constants $c_{B}^{\iota}(j)$ exist such that

$$
\begin{array}{r}
\lambda_{\min }\left(\frac{1}{2}\left(\underline{B}^{\iota}(j)+\bar{B}^{\iota}(j)\right)\right)-\rho\left(\frac{1}{2}\left(\bar{B}^{\iota}(j)-\underline{B}^{\iota}(j)\right)\right) \equiv c_{B}^{\iota}(j) \\
\text { for } j=1, . ., \bar{j}_{\iota}, \iota=1, \ldots, s,
\end{array}
$$

where $\lambda_{\min }$ and $\rho$ denotes the minimal eigenvalue and the spectral radius, respectively. Next, we define the set of admissible matrices

$$
\begin{aligned}
U_{a d}^{\kappa^{\iota}}=\{ & (N \times N)-\text { symmetric matrices } \kappa^{\iota}: \\
& \left.\underline{\kappa}_{i k}^{\iota}(j) \leq\left.\kappa_{i k}^{\iota}\right|_{\Omega_{j}^{\iota}}=\text { const. } \leq \bar{\kappa}_{i k}^{\iota}(j), j \leq \bar{j}_{\iota}, i, k \leq N\right\}
\end{aligned}
$$

where $\underline{\kappa}^{\iota}(j)$ and $\bar{\kappa}^{\iota}(j)$ are given $(N \times N)$ symmetric matrices, $j=1, \ldots, \bar{j}_{\iota}, \iota=$ $1, \ldots, s$. Assume that positive constants $c_{\kappa}^{\iota}(j)$ exist such that

$$
\lambda_{\min }\left(\frac{1}{2}\left(\underline{\kappa}^{\iota}(j)+\bar{\kappa}^{\iota}(j)\right)\right)-\rho\left(\frac{1}{2}\left(\bar{\kappa}^{\iota}(j)-\underline{\kappa}^{\iota}(j)\right)\right) \equiv c_{\kappa}^{\iota}(j) \text { for } j \leq \bar{j}_{\iota}, \iota \leq s,
$$

where $\lambda_{\min }$ and $\rho$ denotes the minimal eigenvalue and the spectral radius, respectively. Then the matrices $\kappa^{\iota}(j)=\left.\kappa^{\iota}\right|_{\Omega_{j}^{\iota}}$ are positive definite for any $\kappa^{\iota} \in U_{a d}^{\kappa^{\iota}}, \iota \leq s, \quad j \leq \bar{j}_{\iota}$.

Now, let us introduce

$$
U_{a d}^{F_{i}^{\iota}}=\left\{f \in L^{\infty}(\Omega): \underline{F}_{i}^{\iota}(j) \leq\left. f\right|_{\Omega_{j}^{\iota}}=\text { const. } \leq \bar{F}_{i}^{\iota}(j), j \leq \bar{j}_{\iota}\right\},
$$

for $i \leq N, \iota \leq s$, where $\underline{F}_{i}^{\iota}(j)$ and $\bar{F}_{i}^{\iota}(j)$ are given constants;

$$
U_{a d}^{W^{\iota}}=\left\{w \in L^{\infty}(\Omega): \underline{W}^{\iota}(j) \leq\left. w\right|_{\Omega_{j}^{\iota}}=\text { const. } \leq \bar{W}^{\iota}(j), j \leq \bar{j}_{\iota}\right\},
$$

for $\iota \leq s$, where $\underline{W}^{\iota}(j)$ and $\bar{W}^{\iota}(j)$ are given constants;

$$
U_{a d}^{T_{1}}=\left\{\mathcal{T} \in L^{\infty}\left(\Gamma_{\tau}\right): \underline{T}_{1}(\iota) \leq\left.\mathcal{T}\right|_{\Gamma_{\tau}^{\iota}}=\text { const. } \leq \bar{T}_{1}(\iota), \iota \leq s\right\},
$$

where $\underline{T}_{1}(\iota)$ and $\bar{T}_{1}(\iota)$ are given constants;

$$
U_{a d}^{u_{0 i}}=\left\{u \in L^{\infty}\left(\Gamma_{u}\right): \underline{u}_{0 i}(\iota) \leq\left. u\right|_{\Gamma_{u}^{\iota}}=\text { const. } \leq \bar{u}_{0 i}(\iota), \iota \leq s\right\},
$$

where $\underline{u}_{0 i}(\iota)$ and $\bar{u}_{0 i}(\iota), i=1, . ., N$, are given constants;

$$
U_{a d}^{P_{i}}=\left\{p \in L^{\infty}\left(\Gamma_{\tau}\right): \underline{P}_{i}(\iota) \leq\left. p\right|_{\Gamma_{\tau}^{\iota}}=\text { const. } \leq \bar{P}_{i}(\iota), \iota \leq s\right\},
$$


where $\underline{P}_{i}(\iota)$ and $\bar{P}_{i}(\iota), i=1, \ldots, N$ are given constants;

$$
U_{a d}^{\beta_{i}^{\iota}}=\left\{b \in L^{\infty}(\Omega): \underline{\beta}_{i}^{\iota}(j) \leq\left. b\right|_{\Omega_{j}^{\iota}}=\text { const. } \leq \overline{\beta^{\iota}}(j), j \leq \bar{j}_{\iota}\right\},
$$

for $i \leq j_{N}, \iota \leq s$, where $\underline{\beta}_{i}^{\iota}(j)$ and $\bar{\beta}_{i}^{\iota}(j)$ are given constants;

$$
\begin{gathered}
U_{a d}^{g^{k l}}=\left\{g \in L^{\infty}\left(\Gamma^{k l}\right):\left.g\right|_{\bar{\Gamma}_{q}^{k l}} \in C^{(0), 1}\left(\bar{\Gamma}_{q}^{k l}\right) ; 0 \leq g(s) \leq \bar{g}_{q}^{k l},\right. \\
\left.|d g / d s| \leq C_{g}^{k l} \text { a.e. in } \Gamma_{q}^{k l}, q \leq Q_{k l}\right\},
\end{gathered}
$$

for all pairs $k, l$ under consideration, where $\bar{g}_{q}^{k l}$ and $C_{g}^{k l}$ are given positive constants. Here $C^{(0), 1}$ denotes the space of Lipschitz-continuous functions.

Finally, we introduce the set of admissible data, as follows:

$$
\begin{aligned}
U_{a d}= & \sqcap_{\iota \leq s} U_{a d}^{B^{\iota}} \times \sqcap_{\iota \leq s} U_{a d}^{\kappa^{\iota}} \times \Pi_{\iota \leq s, j \leq N} U_{a d}^{F_{i}^{\iota}} \times \sqcap_{\iota \leq s} U_{a d}^{W^{\iota}} \times \\
& \times U_{a d}^{T_{1}} \times \Pi_{\iota \leq s, i \leq N} U_{a d}^{\beta^{\iota}} \times \Pi_{i \leq N} U_{a d}^{P_{i}} \times \sqcap_{i \leq N} U_{a d}^{u_{0 i}} \times \sqcap_{k, l} U_{a d}^{g^{k l}} .
\end{aligned}
$$

To obtain $T_{1} \in W_{1}$, we have to extend the boundary values $T_{1} \in U_{\text {ad }}^{T_{1}}$ into the domains $\Omega^{\iota}$ properly, i.e. satisfying the conditions $T_{1}^{k}=T_{1}^{l}$ on all $\Gamma^{k l}$. As a consequence at some intersections $\Gamma^{k l} \cap \bar{\Gamma}_{\tau}$ (if any), additional continuity conditions are necessary in the definition of $U_{a d}^{T_{1}}$. An analogous remark holds for the data $u_{0 i} \in U_{a d}^{u_{0 i}}$ and $\Gamma^{k l} \cap \bar{\Gamma}_{u}$.

Definition 4.1 Instead of the bilinear forms and functionals $b(T, z), a(\mathbf{u}, \mathbf{v})$, $j_{g}(\mathbf{v}), s(z), S(\mathbf{v}, T)$ introduced in Definitions 2.1 and 2.2, we will write $b(A ; T, z)$, $a(A ; \mathbf{u}, \mathbf{v}), j_{g}(A ; \mathbf{v}), s(A ; z), S(A ; \mathbf{v}, T)$ for any $A \in U_{a d}$.

Lemma 4.1 There exist positive constants $c_{i}, i=0,1, \ldots, 6$ independent of $A \in U_{a d}$, such that

$$
\begin{gathered}
b(A ; z, z) \geq C_{0}\|z\|_{W^{1}}^{2} \quad \forall z \in V_{1} \\
|b(A ; z, y)| \leq C_{1}\|z\|_{W^{1}}\|y\|_{W^{1}} \quad \forall z, y \in W_{1} \\
a(A ; \mathbf{v}, \mathbf{v}) \geq C_{2}\|\mathbf{v}\|_{W}^{2} \quad \forall \mathbf{v} \in V \\
|a(A ; \mathbf{v}, \mathbf{w})| \leq C_{3}\|\mathbf{v}\|_{W}\|\mathbf{w}\|_{W} \quad \forall \mathbf{v}, \mathbf{w} \in W \\
|s(A ; z)| \leq C_{4}\|z\|_{0, \Omega} \quad \forall z \in V_{1} \\
|S(A ; \mathbf{v}, T)| \leq C_{5}\left(\|\mathbf{v}\|_{0, \Omega}+\|\mathbf{v}\|_{0, \Gamma_{\tau}}+\left\|T-T_{0}\right\|_{0, \Omega}\|\mathbf{v}\|_{W}\right) \quad \forall \mathbf{v}, \mathbf{w} \in W \\
\left|j_{g}(A ; \mathbf{u})-j_{g}(A ; \mathbf{v})\right| \leq C_{6} \sum_{\iota \leq s}\left\|\mathbf{u}^{\iota}-\mathbf{v}^{\iota}\right\|_{0, \partial \Omega^{\iota}} \quad \forall \mathbf{u}, \mathbf{v} \in W
\end{gathered}
$$


Proof: By Theorem 5 in (Rohn (1994)), we have

$$
\lambda_{\min }\left(\kappa^{\iota}(j)\right) \geq c_{\kappa}^{\iota}(j) \forall \kappa^{\iota} \in U_{a d}^{\kappa^{\iota}}, \iota \leq s, j \leq \bar{j}_{\iota} .
$$

As a consequence, we obtain

$$
b(A ; z, z) \geq\left(\min _{\iota \leq s, j \leq \bar{j}_{\iota}} c_{\kappa}^{\iota}(j)\right) \sum_{\iota \leq s} \int_{\Omega^{\iota}}\left|\operatorname{grad} z^{\iota}\right|^{2} d \mathbf{x} .
$$

Then we have

$$
\int_{\Omega^{\iota}}\left|\operatorname{grad} z^{\iota}\right|^{2} d \mathbf{x} \geq C_{1}^{\iota}\left\|z^{\iota}\right\|_{1, \Omega^{\iota}}^{2}
$$

for any restriction $z^{\iota}$ of $z \in V_{1}$. Combining (4.24) and (4.25), we arrive at (4.17).

The inequality (4.18) follows from the definitions of $U_{a d}^{\kappa^{\iota}}$ immediately.

Arguing as in (4.24), we may write

$$
a(A ; \mathbf{v}, \mathbf{v}) \geq\left(\min _{\iota \leq s, j \leq \bar{j}_{\iota}} c_{B}^{\iota}(j)\right) \sum_{\iota \leq s} \int_{\Omega^{\iota}} \sum_{k=1}^{j_{N}} e_{k}^{2}\left(\mathbf{v}^{\iota}\right) d \mathbf{x}
$$

The Korn's inequality

$$
\int_{\Omega^{\iota}} e\left(\mathbf{v}^{\iota}\right): e\left(\mathbf{v}^{\iota}\right) d \mathbf{x} \geq C_{2}^{\iota}\left\|\mathbf{v}^{\iota}\right\|_{1, \Omega^{\iota}}^{2}
$$

holds for any restriction $\mathbf{v}^{\iota}$ of $\mathbf{v} \in V$. Since

$$
\frac{1}{2} e: e \leq \sum_{k=1}^{j_{N}} e_{k}^{2}
$$

(recall the formula (2.14)), combining (4.26)-(4.28), we obtain the inequality (4.19). The inequality (4.20) is an easy consequence of the definitions of $U_{a d}^{B^{\iota}}$.

Thus, we may write

$$
|s(A ; z)| \leq \sum_{\iota \leq s}\left(\max _{j \leq \bar{j}_{\iota}}\left|\bar{W}^{\iota}(j)\right|\right) \int_{\Omega^{\iota}}\left|z^{\iota}\right| d \mathbf{x} \leq C_{4}\|z\|_{0, \Omega}
$$

Next, we have

$$
\begin{aligned}
|S(A ; \mathbf{v}, T)| \leq & \sum_{\iota \leq s}\left(N^{\frac{1}{2}}\left(\max \left\{\left|\bar{F}_{i}^{\iota}(j)\right|,\left|\underline{F}_{i}^{\iota}(j)\right|\right\}\right)\left\|\mathbf{v}^{\iota}\right\|_{0, \Omega^{\iota}}+\right. \\
& +C\left(\max \bar{\beta}^{\iota}(j)\right) \int_{\Omega^{\iota}} 2\left|T^{\iota}-T_{0}^{\iota}\right|\left\|e\left(\mathbf{v}^{\iota}\right)\right\| d \mathbf{x}+ \\
& \left.+N^{\frac{1}{2}}\left(\max \left\{\left|\bar{P}_{i}(\iota)\right|,\left|\underline{P}_{i}(\iota)\right|\right\}\right)\left\|\mathbf{v}^{\iota}\right\|_{0, \Gamma_{\tau}^{\iota}}\right) \leq \\
\leq & C_{5}\left(\|\mathbf{v}\|_{0, \Omega}+\|\mathbf{v}\|_{0, \Gamma_{\tau}}+\left\|T-T_{0}\right\|_{0, \Omega}\|\mathbf{v}\|_{W}\right) .
\end{aligned}
$$


Finally, we may write

$$
\begin{aligned}
\mid j_{g}(A ; \mathbf{u})-j_{g}(A ; \mathbf{v}) & \leq \sum_{k, l} \int_{\Gamma^{k l}} g^{k l}\left|\left(\mathbf{u}_{t}^{k}-\mathbf{v}_{t}^{k}\right)-\left(\mathbf{u}_{t}^{l}-\mathbf{v}_{t}^{l}\right)\right| d s \leq \\
& \leq \sum_{k, l} \max _{q \leq Q_{k l}} g_{q}^{k l} \int_{\Gamma^{k l}}\left|\left(\mathbf{u}_{t}^{k}-\mathbf{v}_{t}^{k}\right)-\left(\mathbf{u}_{t}^{l}-\mathbf{v}_{t}^{l}\right)\right| d s \leq \\
& \leq C \sum_{k, l} \sum_{i \leq N}\left(\left\|u_{i}^{k}-v_{i}^{k}\right\|_{0, \Gamma^{k l}}+\left\|u_{i}^{l}-v_{i}^{l}\right\|_{0, \Gamma^{k l}}\right) \leq \\
& \leq C_{6} \sum_{\iota \leq s}\left\|\mathbf{u}^{\iota}-\mathbf{v}^{\iota}\right\|_{0, \partial \Omega^{\iota}} .
\end{aligned}
$$

Proposition 4.2 There exists a unique weak solution $T(A)$ of the problem $\mathcal{P}_{1}$ for any $A \in U_{a d}$ and $\mathbf{u}(A)$ of problem $\mathcal{P}_{2}$ for any $A \in U_{a d}$.

For the proof see Hlaváček, Nedoma (2004).

\subsection{Criteria of worst scenario}

To find the "worst", i.e. the most "dangerous" input data $A$ in the set $U_{a d}$, we need a criterion, i.e. a functional, which depends on the solution $T(A)$ or $\mathbf{u}(A)$ of problem $\mathcal{P}_{1}$ or $\mathcal{P}_{2}$, respectively.

Next, we present several examples of such criteria.

Let $G_{r} \subset \Omega, r=1, \ldots, \bar{r}$, be (small) subdomains, adjacent to the boundaries $\partial \Omega^{\iota}$, for example. We can define

$$
\Phi_{1}(T)=\max _{r \leq \bar{r}} \varphi_{r}(T)
$$

where $\varphi_{r}(T)=\left(\operatorname{meas}_{N} G_{r}\right)^{-1} \int_{G_{r}} T d \mathbf{x}$;

let $G_{r}^{\prime} \subset \Gamma_{u}, r \leq \bar{r}$ and

$$
\Phi_{2}(T)=\max _{r \leq \bar{r}} \psi_{r}(T)
$$

where $\psi_{r}(T)=\left(\text { meas }_{N-1} G_{r}^{\prime}\right)^{-1} \int_{G_{r}^{\prime}} T d s$.

Next, we define

$$
\Phi_{3}(\mathbf{u})=\max _{r \leq \bar{r}} \chi_{r}(\mathbf{u})
$$

where $\chi_{r}(\mathbf{u})=\left(\text { meas }_{N} G_{r}\right)^{-1} \int_{G_{r}} u_{i} n_{i}\left(X_{r}\right) d \mathbf{x}$; where $\mathbf{n}\left(X_{r}\right)$ is the unit outward normal at a fixed point $X_{r} \in \partial \Omega^{\iota} \cap \partial G_{r}$ (if $G_{r} \subset \Omega^{\iota}$ ) to the boundary $\partial \Omega^{\iota}$;

$$
\Phi_{4}(\mathbf{u})=\max _{r \leq \bar{r}} \chi_{r}^{\prime}(\mathbf{u})
$$

where $\chi_{r}^{\prime}(\mathbf{u})=\left(\text { meas }_{N-1} G_{r}^{\prime}\right)^{-1} \int_{G_{r}^{\prime}} u_{i} n_{i}\left(X_{r}\right) d s ; G_{r}^{\prime} \subset \cup_{\iota \leq s} \partial \Omega^{\iota} \backslash \Gamma_{u}$. 
Since the weak solution $\mathbf{u}(A)$ of our problem (2.15) depends on $T(A)$, then $\mathbf{u}(A)=\mathbf{u}(A ; T(A))$ and instead of $\Phi_{i}(\mathbf{u})$ we write $\Phi_{i}(A ; \mathbf{u}, T)$. Another choice is

$$
\Phi_{5}(A ; \mathbf{u}, T)=\max _{r \leq \bar{r}} \omega_{r}(A ; \mathbf{u}, T)
$$

where $\omega_{r}(A ; \mathbf{u}, T)=\left(\text { meas }_{N} G_{r}\right)^{-1} \int_{G_{r}} I_{2}^{2}(\tau(A ; \mathbf{u}, T)) d \mathbf{x}$.

Here $I_{2}(\tau)$ denotes the intensity of shear stress (see, e.g. Nečas, Hlaváček $(1981)$ ), i.e. the second fundamental invariant of the stress tensor deviator $\tau^{D}$, i.e.

$$
\begin{gathered}
I_{2}^{2}(\tau)=\sum_{i, j=1}^{3} \tau_{i j}^{D} \tau_{i j}^{D}, \quad \tau_{i j}^{D}=\tau_{i j}-\frac{1}{3} \tau_{k k} \delta_{i j} ; \\
I_{2}^{2}=\frac{2}{3}\left[\tau_{11}^{2}+\tau_{22}^{2}+\tau_{33}^{2}-\left(\tau_{11} \tau_{22}+\tau_{11} \tau_{33}+\tau_{22} \tau_{33}\right)+3\left(\tau_{12}^{2}+\tau_{13}^{2}+\tau_{23}^{2}\right)\right] \text { for } N=3 .
\end{gathered}
$$

In (4.33), $\tau(A ; \mathbf{u}, T)$ is defined by the formula (2.7). For orthotropic material and plane strain, we have to insert $\tau_{13}=\tau_{23}=0$.

If the friction can be neglected (as in Hlaváček, Nedoma (2002b), Nečas, Hlaváček (1981), Nedoma (1998)), we set $g_{c}^{k l} \equiv 0$ and define e.g.

$$
\Phi_{6}(A ; \mathbf{u}, T)=\max _{r \leq \bar{r}} \mu_{r}(A ; \mathbf{u}, T)
$$

$\mu_{r}(A ; \mathbf{u}, T)=\left(\text { meas }_{N} G_{r}\right)^{-1} \int_{G_{r}}\left(-\tau_{n}(A ; \mathbf{u}, T)\right) d \mathbf{x}$; and $G_{r}$ is a small subdomain adjacent to $\Gamma_{c}$.

Now we formulate the worst scenario problems as follows:

find

$$
A^{0 i}=\arg \max _{A \in U_{a d}} \Phi_{i}(T(A)), i=1,2,
$$

and

$$
A^{0 i}=\arg \max _{A \in U_{a d}} \Phi_{i}(\mathbf{u}(A), T(A)), i=3,4,5,6,
$$

where $T(A)$ are $\mathbf{u}(A)$ are weak solutions of the problem $\mathcal{P}_{1}$ and $\mathcal{P}_{2}$, respectively.

Remark 4.1 Since the weak solution $\mathbf{u}(A)$ of problem $\mathcal{P}_{2}$ depends on $T(A)$, $\mathbf{u}(A) \equiv \mathbf{u}(A ; T(A))$ and we write $\Phi_{i}(\mathbf{u}(A), T(A))$, instead of $\Phi_{i}(\mathbf{u}(A))$ for $i=3,4$ in $(4.36)$.

\subsection{Stability of weak solutions}

To analyze the solvability of worst scenario problems $(4.35),(4.36)$ we have to study the mapping $A \mapsto T(A)$ and $A \mapsto \mathbf{u}(A, T(A))$. First, we introduce the following decomposition of $A \in U_{a d}: A=\left\{A^{\prime}, A^{\prime \prime}\right\}$, where

$$
A^{\prime}=\left\{\Pi_{\iota \leq s} \Pi_{j \leq \bar{j}_{\iota}} \kappa^{\iota}(j), \sqcap_{\iota \leq s} \Pi_{j \leq \bar{j}_{\iota}} W^{\iota}(j), \sqcap_{\iota \leq s} T_{1}^{\iota}\right\}, \quad A^{\prime} \in \mathbb{R}^{p_{1}},
$$




$$
p_{1}=\left(j_{N}+1\right) \sum_{\iota \leq s} \bar{j}_{\iota}+s
$$

and

$$
\begin{gathered}
A^{\prime \prime}=\left\{\sqcap_{\iota \leq s} \sqcap_{j \leq \bar{j}_{\iota}} B^{\iota}(j), \sqcap_{\iota \leq s} \sqcap_{j \leq \bar{j}_{\iota}} \mathbf{F}^{\iota}(j), \sqcap_{\iota \leq s} \mathbf{P}^{\iota},\right. \\
\left.\prod_{\iota \leq s} \mathbf{u}_{0}^{\iota}, \sqcap_{\iota \leq s} \sqcap_{j \leq \bar{j}_{\iota}} \beta^{\iota}(j), \sqcap_{k, l} \sqcap_{q \leq Q_{k l}} g^{k l}(q)\right\}, \\
A^{\prime \prime} \in \mathbb{R}^{p_{2}} \times \sqcap_{k, l} \sqcap_{q \leq Q_{k l}} C\left(\bar{\Gamma}_{q}^{k l}\right), \quad p_{2}=\left(\sum_{\iota \leq s} \bar{j}_{\iota}\right)\left[\left(3+j_{N}\right) j_{N} / 2+N(1+2 s)\right] .
\end{gathered}
$$

We are going to show the continuity of the mappings $A^{\prime} \mapsto T\left(A^{\prime}\right)$ for $A^{\prime} \in$ $U_{a d}^{\prime}=\Pi_{\iota \leq s} U_{a d}^{\kappa^{\iota}} \times \Pi_{\iota \leq s} U_{a d}^{W^{\iota}} \times U_{a d}^{T_{1}^{\iota}}$ and $A \mapsto \mathbf{u}\left(A, T\left(A^{\prime}\right)\right)$ for $A^{\prime \prime} \in U_{a d}^{\prime \prime}=$ $\Pi_{\iota \leq s} U_{a d}^{B^{\iota}} \times \Pi_{\iota \leq s, j \leq N} U_{a d}^{F^{\iota}} \times \Pi_{\iota \leq s, i \leq N} U_{a d}^{\beta^{\iota}} \times \Pi_{i \leq N} U_{a d}^{P_{i}} \times \Pi_{i \leq N} U_{a d}^{u_{0 i}}$, respectively. Since the problem discussed is quasi-coupled, we have the following theorem and lemma:

Lemma 4.3 If $A_{n} \in U_{a d}, A_{n} \rightarrow A$ in $U$, where $U=\mathbb{R}^{p_{1}+p_{2}} \times \sqcap_{k, l} \sqcap_{q \leq Q_{k l}} C\left(\bar{\Gamma}_{q}^{k l}\right)$, and $\mathbf{u}_{n} \rightarrow \mathbf{u}$ weakly in $W$ then

$$
\begin{gathered}
a\left(A_{n} ; \mathbf{u}_{n}, \mathbf{v}\right) \rightarrow a(A ; \mathbf{u}, \mathbf{v}) \quad \forall \mathbf{v} \in W \\
S\left(A_{n} ; \mathbf{u}_{n}, T\right) \rightarrow S(A ; \mathbf{u}, T) \quad \forall T \in W_{1}, \\
j_{g}\left(A_{n} ; \mathbf{u}\right) \rightarrow j_{g}(A ; \mathbf{u}) .
\end{gathered}
$$

For the proof see Hlaváček, Nedoma (2004).

Theorem 4.4 Let $A^{\prime} \in U_{a d}^{\prime}, A_{n}^{\prime} \rightarrow A^{\prime}$ in $\mathbb{R}^{p_{1}}$ as $n \rightarrow \infty$. Then

$$
T\left(A_{n}^{\prime}\right) \rightarrow T\left(A^{\prime}\right) \quad \text { in } W_{1} .
$$

Let $A_{n} \in U_{a d}, A_{n} \rightarrow A$ in $U \equiv \mathbb{R}^{p_{1}+p_{2}} \times \sqcap_{k, l} \sqcap_{q \leq Q_{k l}} C\left(\bar{\Gamma}_{q}^{k l}\right)$. Then

$$
\mathbf{u}\left(A_{n}\right) \rightarrow \mathbf{u}(A) \quad \text { in } W
$$

For the proof see Hlaváček, Nedoma (2004).

\subsection{Existence of a solution of the worst scenario problem}

To prove the existence of a solution of the worst scenario problem, we will use the following lemma.

Lemma 4.5 Let $\Phi_{i}(T), i=1,2$, be defined by (4.29), (4.30) and let $T_{n} \rightarrow T$ in $W_{1}$, as $n \rightarrow \infty$. Then

$$
\lim _{n \rightarrow \infty} \Phi_{i}\left(T_{n}\right)=\Phi_{i}(T), \quad i=1,2
$$


Let $\Phi_{i}(\mathbf{u}), i=3,4$, be defined by (4.31) and (4.32) and let $\mathbf{u}_{n} \rightarrow \mathbf{u}$ in $W$, as $n \rightarrow \infty$. Then

$$
\lim _{n \rightarrow \infty} \Phi_{i}\left(\mathbf{u}_{n}\right)=\Phi_{i}(\mathbf{u}), \quad i=3,4 .
$$

Let $\Phi_{i}(A ; \mathbf{u}, \mathbf{T}), i=5,6$, be defined by (4.33) and (4.34). Let $A_{n} \rightarrow A$ in $U$, $A_{n} \in U_{a d}, \mathbf{u}_{n} \rightarrow \mathbf{u}$ in $W$ and $T_{n} \rightarrow$ Tin $L^{2}(\Omega)$. Then

$$
\lim _{n \rightarrow \infty} \Phi_{i}\left(A_{n}, \mathbf{u}_{n}, T_{n}\right)=\Phi_{i}(A, \mathbf{u}, T), i=5,6
$$

The main result gives the next theorem:

Theorem 4.6 There exists at least one solution of the worst scenario problems (4.35), (4.36), $i=1, \ldots, 6$.

Proof: Let us denote

$$
J_{i}(A)=\Phi_{i}(T(A)), \quad i=1,2 .
$$

If $A_{n} \in U_{a d}, A_{n} \rightarrow A$ in $U$ as $n \rightarrow \infty$, then $A_{n}^{\prime} \rightarrow A$ in $\mathbb{R}^{p_{1}}$ and $T\left(A_{n}\right) \rightarrow T(A)$ in $W_{1}$ by virtue of Theorem 4.4. Using Lemma 4.5, we obtain

$$
J_{i}\left(A_{n}\right) \rightarrow J_{i}(A)
$$

so that $J_{i}$ is continuous on the set $U_{a d}$.

It is easy to show that $U_{a d}$ is compact subset of $U$, if we employ Arzela-Ascoli Theorem for $U_{a d}^{g^{k l}}$.

As a consequence, $J_{i}$ attains its maximum on $U_{a d}$.

The same argument can be applied to

$$
J_{i}(A)=\Phi_{i}(A ; \mathbf{u}(A) T(A)), \quad i=3,4,5,6 .
$$

Here we employ Theorem 4.4 and Lemma 4.5 to verify the continuity of $J_{i}$ on the set $U_{a d}$.

\section{$5 \quad$ Numerical experiments}

For approximations of the problem we can employ the finite element method and the algorithm of Section 3 based on the nonoverlapping domain decomposition approach developed in Daněk, Hlaváček, Nedoma (2004). 
The geomechanical model problem describing a loaded tunnel which is crossing by a deep fault and based on the geomechanical theory and models having connection with radioactive waste repositories (Nedoma (1998)). A geometry of the problem is in Fig. 1.

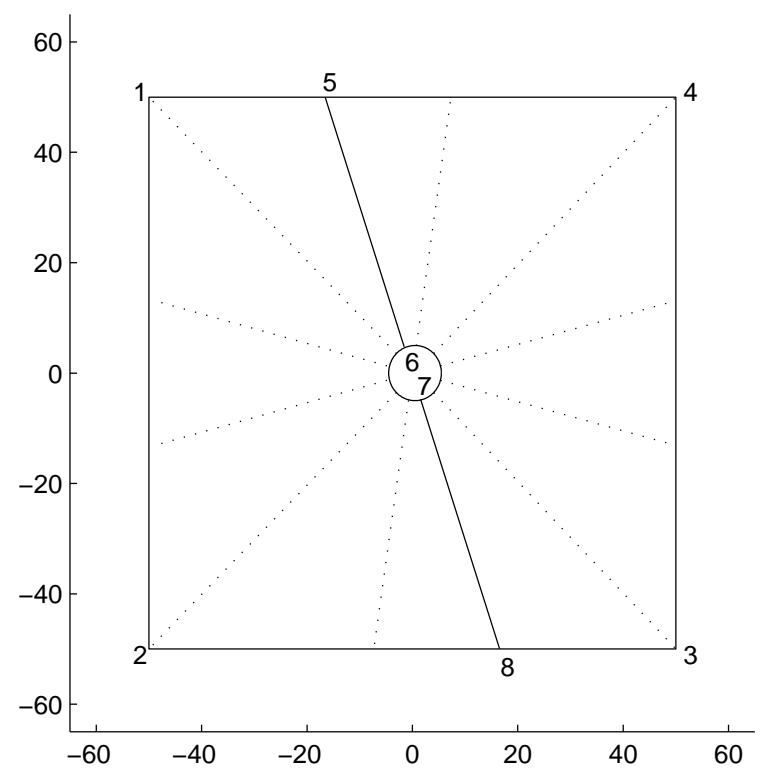

FiguRE 1. Geometry of the problem.

Material parameters: 2 regions with Young's modulus $E=5,2 \times 10^{9}[\mathrm{~Pa}]$ and Poisson's ratio $\nu=0,18$. Specific gravity is $2,45 \times 10^{4}[\mathrm{~Pa} / \mathrm{m}]$.

Boundary conditions: Prescribed displacement $\left(2,5 \times 10^{-2} ; 0\right)[\mathrm{m}]$ on $1-2$. Pressure $0,5 \times 10^{7}[\mathrm{~Pa}]$ on $1-4$ and $2-8$ and $1 \times 10^{7}[\mathrm{~Pa}]$ on $8-3$. Bilateral contact boundary on 3-4. Unilateral contact boundary: 5-6 and 7-8. Given slip limit is $10^{6}[\mathrm{~Pa}]$. Zero surface forces on the tunnel wall.

Discretization statistics: 12 subdomains, 5501 nodes, 9676 elements, 10428 unknowns, 89 unilateral contact conditions, 466 interface elements.

Convergence statistics: 21 iterations of the PCG algorithm for the auxiliary problem, 15 iterations of the successive approximations method for accuracy $10^{-6}$, total 39 iterations of the PCG algorithm for the original problem.

Fig. 2 represents detail of deformations and Fig. 3 shows displacements in a neighbourhood of the tunnel. On Figs. 4 and 5 details of principal stresses are displayed in a neighbourhood of the tunnel. 


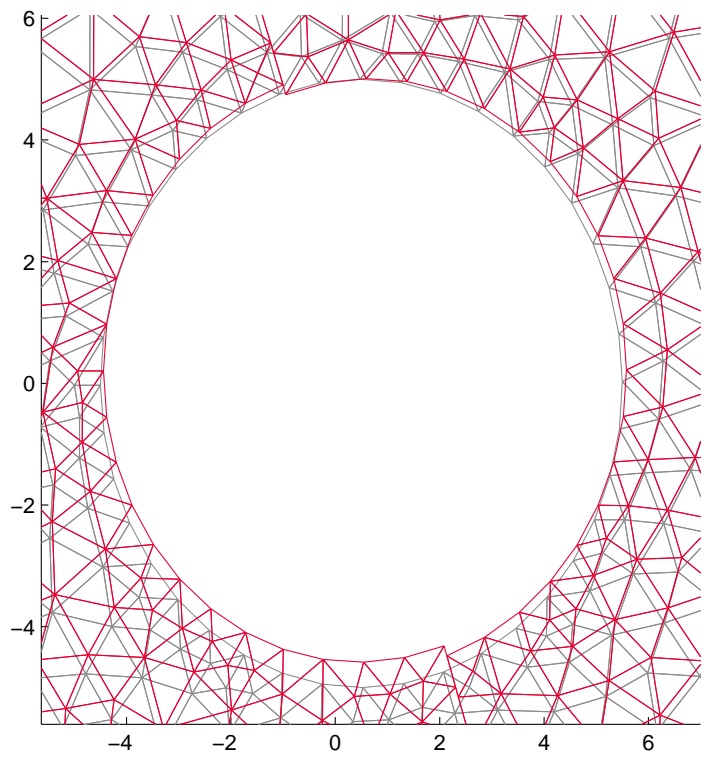

FiguRE 2. Detail of deformations (enlarging factor is 10).

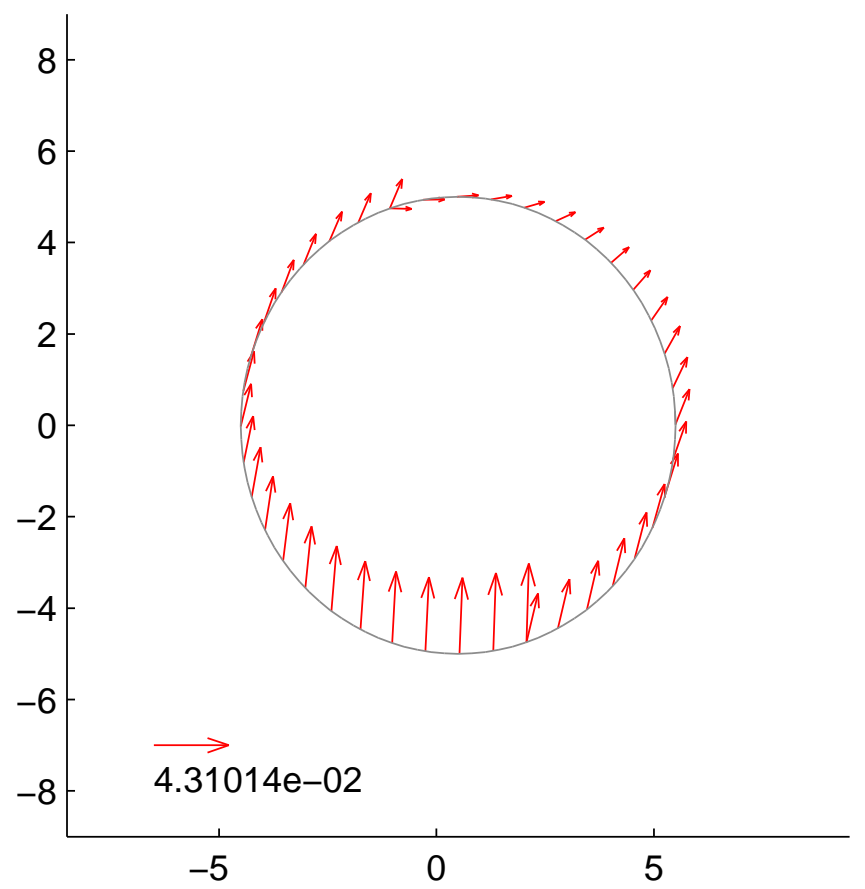

Figure 3. Detail of displacements on the tunnel wall. 


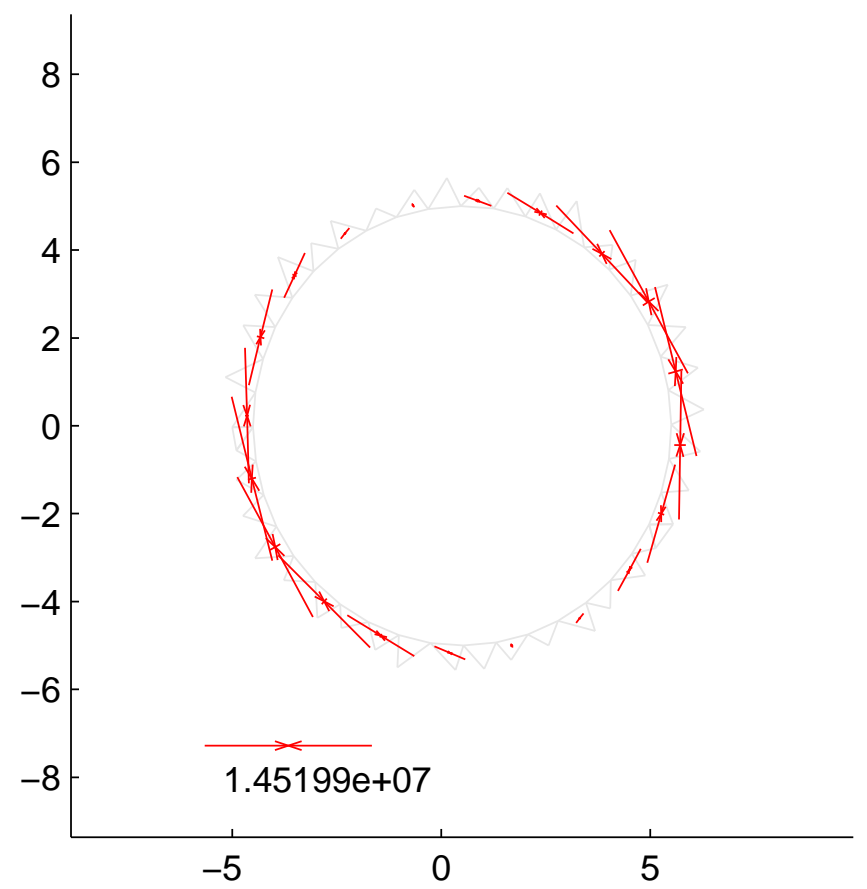

Figure 4. Detail of principal stresses on the tunnel wall.

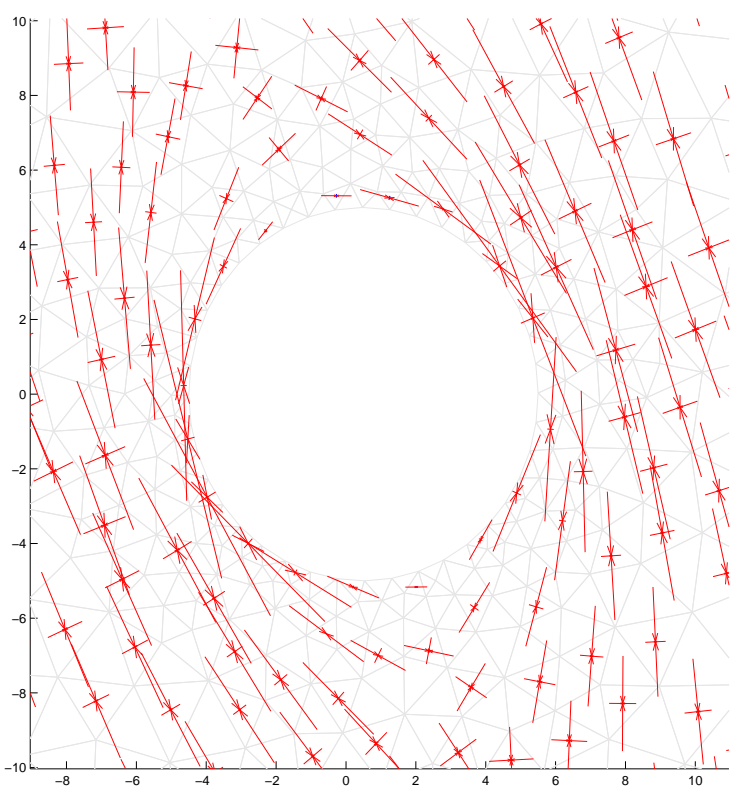

FigURE 5. Detail of principal stresses in a neighbourhood of the tunnel. 


\section{Conclusions}

The theory presented in this paper represents extension of geomechanical problems solved in Nedoma (1987), (1998) for the case if input data, i.e. thermal conductivity and elastic coefficients, body and surface forces, thermal sources, body and surface forces, coefficients of thermal expansion, boundary values, coefficient of friction on contact boundaries, etc. are uncertain. Since the theory is an extension of problems solved in Nedoma (1998) it can be used for mathematical models connected with the safety of construction and of operation of the radioactive waste repositories. The models involve input data (as thermal conductivity and elastic coefficients, body and surface forces, thermal sources, coefficients of thermal expansion, boundary values, coefficient of friction on contact boundaries, etc.) which cannot be determined uniquely, but only in some intervals, given by the accuracy of measurements and the approximate solutions of identification problems. The "reliable solution" denotes the worst case among a set of possible solutions where the degree of badness is measured by a criterion functional. For the safety of the high level radioactive waste repositories and other structures under critical conditions we seek the maximal value of this functional, which depends on the solution of the mathematical model. Then for the computations of such problems (some mean values of temperatures, displacements, intensity of shear stresses, principal stresses, stress tensor components, normal and tangential components of the displacement or stress vector on the contact boundaries, etc.) we have to formulate a corresponding maximization (worst scenario) problem. Then methods and algorithms known from "optimal design" can be used.

To construct a model of structures under the influence of critical conditions the influence of global tectonics onto a local area, where the critical structure is built as well as the influence of the resulting local geomechanical processes on a critical structure must be taken into account (Nedoma (1998)). Problems of this kind with uncertain input data are problems with high level radioactive waste repositories. In the case of the high level radioactive waste repositories the effects of geodynamical processes in the sense of plate tectonics must be taken into consideration, namely in regions near tectonic areas (e.g. the Japan island arc, the Central and South Europe, etc.), but also in the platform regions (as in Sweden, Canada, etc.). But in geomechanics and geodynamics our information about input data are very questionable as we obtain input data with very small accuracy. Therefore, the presented method as well as algorithms used give a worst scenario (anti-optimal) solution of the problem studied. In the practice it represents a tolerance solution corresponding to the structures in critical situations and, in fact, the obtained solutions facilitate to ensure the high security of constructions and operations of structures under critical situation (e.g. high level radioactive waste repositories). Another example is represented by modelling an interaction between a tunnel wall and 
a rock massif in the radioactive waste repository tunnels or by modelling of a tunnel crossing by an active deep fault(s), respectively.

The parallel algorithm presented in this paper is based on the nonoverlapping domain decomposition method developed in Daněk, Hlaváček, Nedoma (2004). The algorithm is derived from the primal formulation in displacement, uses grouping every two subdomains which share a contact area into a single "nonlinear" subdomain and follows the approach proposed by Le Tallec (1994) for linear problems. Other possible variants are to consider mixed formulation involving both displacements and stresses or dual formulation eliminating the displacement unknowns from mixed problem.

\section{Acknowledgements}

The research was supported by the grant COPERNICUS-HIPERGEOS II KIT 977006 and grants of the Ministry of Education, Youth and Sport of the Czech Republic No. OK-407 and MSM 235200001.

\section{References}

[1] Daněk, J. (2002). Domain decomposition algorithm for solving contact of elastic bodies. In: Sloot, P.M.A. et al. (Eds), Proc. of the ICCS'2002, Springer Lecture Notes in Computer Sciences 2331, 820-829, Berlin, Heidelberg.

[2] Hlaváček, I., (1999). Domain decomposition applied to a unilateral contact of elastic bodies. Technical Report No 785, ICS AS CR, Prague.

[3] Hlaváček, I. (1999). Reliable solution of a Signorini contact problem with friction, considering uncertain data. Numer. Linear Algebra Appl. 6, 411-434.

[4] Hlaváček, I., Nedoma, J. (2002). On a solution of a generalized semicoercive contact problem in thermo-elasticity. Mathematics and Computers in Simulation, 60, 1-17.

[5] Hlaváček, I., Nedoma, J. (2002b). Reliable solution of a unilateral frictionless contact problem in quasi-coupled thermo-elasticity with uncertain input data. In: Sloot, P.M.A. et al. (Eds), Proc. of the conference ICCS'2002, Springer Lecture Notes in Computer Science 2331, 840-851, Berlin, Heidelberg.

[6] Hlaváček, I., Nedoma, J. (2004). Reliable solution of a unilateral contact problem with uncertain input data in thermo-elasticity. Mathematics and Computers in Simulation (to appear). 
[7] Daněk, J., Hlaváček, I., Nedoma, J. (2004). Domain decomposition for generalized unilateral semi-coercive contact problem with friction in elasticity. Numer. Linear Algebra Appl. (to appear).

[8] LeTallec, P. (1994). Domain decomposition methods in computational mechanics. Comput. Mech. Advances 1, 121-220.

[9] Nečas, J., Hlaváček, I. (1981). Mathematical Theory of Elastic and Elastoplastic Bodies: An Introduction. Elsevier, Amsterdam.

[10] Nedoma, J. (1987). On the Signorini problem with friction in linear thermoelasticity. The quasi-coupled 2D-case. Appl.Math. 32(3), 186-199.

[11] Nedoma, J. (1998). Numerical Modelling in Applied Geodynamics. John Wiley\&Sons, Chichester, New York, Weinheim, Brisbane, Singapore, Toronto.

[12] Pavarino, L.F., Toselli, A. (2002). Recent Developments in Domain Decomposition Methods. Springer Vlg., Lect. Notes in Comput. Sci Eng., vol. 23.

[13] Rohn, J. (1994). Positive definiteness and stability of interval matrices. SIAM J. Matrix Anal. Appl. 15, 175-184. 